\title{
Design and Application of TBM Cutter Information Management System
}

\author{
Liu Shang ${ }^{1+}$, Xia Yimin ${ }^{2}$, Fu Jie ${ }^{2}$, Wang Qiufeng ${ }^{3}$, Mao Bin ${ }^{4}$ and Niu Bing ${ }^{4}$ \\ ${ }^{1}$ School of Software, Central South University, ChangSha; 410083, China \\ ${ }^{2}$ College of Mechanical and Electrical Engineering,Central South University, ChangSha; 410083, China \\ ${ }^{3}$ Central South University Library, ChangSha; 410083, China \\ ${ }^{4}$ China Railway 18th Bureau Group Co., Ltd.; 300222, China
}

\begin{abstract}
In view of the problems existing in the cutter management of the full face rock tunnel boring machine (TBM), such as the information is not timely, the information is not accurate and the data storage time is too short. Based on the requirement analysis and function module, the TBM Cutter management information system is established. The system uses the $\mathrm{B} / \mathrm{S}$ mode three layer architecture and through the $\mathrm{C \#}$ language to write the background code to complete the database connection and operation. Finally, the TBM cutter information management system is deployed to a water supply project. The results show that the system has good scalability and versatility.And can realize the cutter monitoring analysis and efficient management.
\end{abstract}

Keywords: ASP.NET, TBM cutter, management information system

\section{Introduction}

With the continuous development of tunnel construction in China, the application of tunnel boring machine (TBM) shows a gradual upward trend. To achieve TBM efficient economic rock breaking is the urgent need to solve the construction of the key issues [1-2].TBM cutter in the tunnel boring machine, with the rotation of the cutter head of the tunnel the surface rock crushing function. Cutter is a key component of great consumption of excavation equipment. How to scientifically manage a large number of tool failure information and inventory information, thereby improving the construction efficiency of TBM is an urgent need to solve the problem.

At present, many industries have already used information management technology to establish relevant information management system for scientific management [3-4]. Renjun proposed TBM integrated management information system of Xinjiang Osaka tunnel project [5]. Okubo S developed an expert evaluation system for assessing the adaptability of TBM through Pascal and Basic languages [6]. These software have great effect on the development of shield tunneling machine, and the development of management information system has achieved great success. However, the management of TBM in China is only aimed at one aspect, such as fault diagnosis and condition monitoring. There is little management of the corresponding information, and the management system is not refined to the details of the important part management of the cutter. It is difficult to form a data resource base to provide basic data for research and analysis.

Based on the actual situation of a water supply project, this paper designs a cutter management information system based on ASP.NET technology, taking into account the specific needs of the manager for

\footnotetext{
+ Corresponding author. Tel.: 13278887787.

E-mail address: 1shang@csu.edu.cn.
} 
cutter management. The main achievement: user authentication, cutter accessories information management, account management, inventory information management, the cutter wear information management, out of warehouse information management, cutter wear, cutter changes, statistics and other functions. The system can ensure the security of the data, it is convenient for management personnel to access and use. The system has been applied to a project TBM project.

\section{System Architecture}

Considering the convenience of the maintenance of the business process and system of enterprise, the system adopts three layer Browser/Server architecture to develop [7]. as shown in Fig. 1 in the system is the DAL (data access layer) and BLL (business logic layer), two layer completely corresponding, plus UI (presentation layer), each layer of clear division of labor and do not influence each other, which not only can clearly show the structure of TBM cutter information management system, at the same time can reduce the maintenance and upgrade of the system of the late difficulty. In addition, we only need to pay attention to the structure of a layer, can improve the efficiency of the whole, and each layer becomes more replacement Easy, the dependence between the layers is lower, more conducive to the standardization of the system and the reuse of the logic layer.
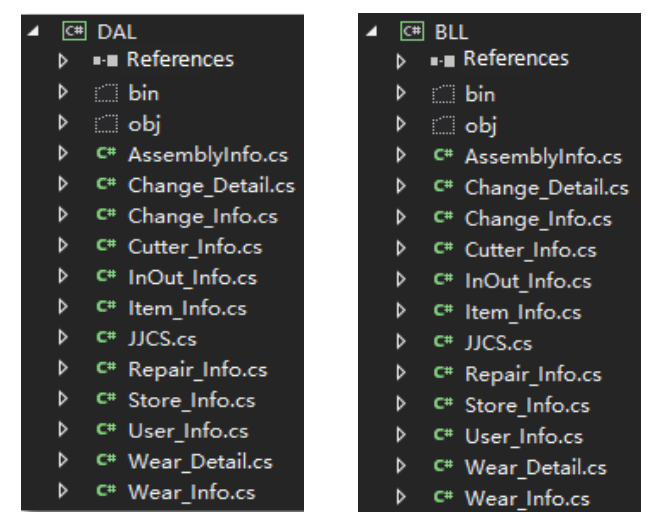

Fig. 1: Data access layer and business logic layer

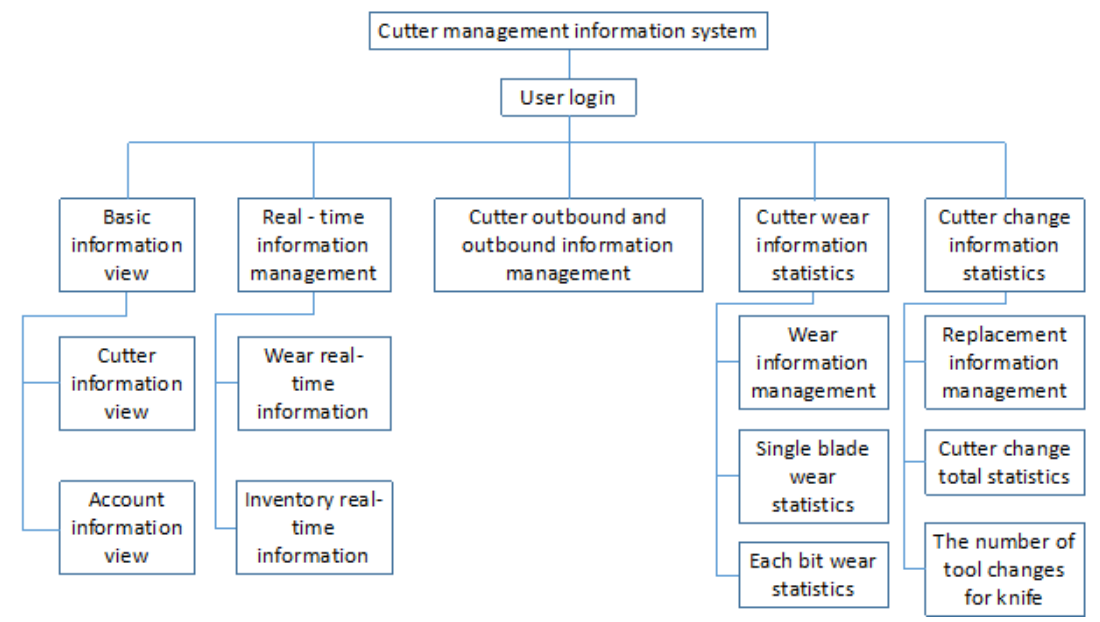

Fig. 2: Function structure of cutter management information system

\section{System Design}

\subsection{Overall system design}

A survey of relevant information management cutter TBM in the process of construction, and the related literature at home and abroad, this paper designs the main function module and the TBM cutter management information system, it not only need to meet the management operational recommendations, clear navigation, but also needs to satisfy the management staff of cutter inventory information, the use of information and related data statistics. Function. At present, the system needs to achieve the main tasks include: user login , basic information, real-time information management, cutter and accessories, Taiwan Account management , 
cutters and accessories, the statistics of cutter wear, the statistics. The number of cutting cutter management information system overall function structure as shown in Fig. 2.

\subsection{System Database Design}

In the cutter management system, personnel management system can be convenient, accurate search and maintain relevant data. The system mainly includes: the management data maintenance personnel identity information, cutter layout information, cutters and spare parts ledger information, cutter inventory information, cutter information storage, information cutter wear and cutter replacement information. Therefore, need according to the above information to establish a database table.

Complete database structure design, need to correlate the relationship between database tables.Such as a knife replacement to the cutter, then exchange information between each cutter and cutter time is one to many relationship. In order to ensure the consistency of the database according to the number, the relationship between the need for further design. Designed the database diagram as shown in Fig. 3.

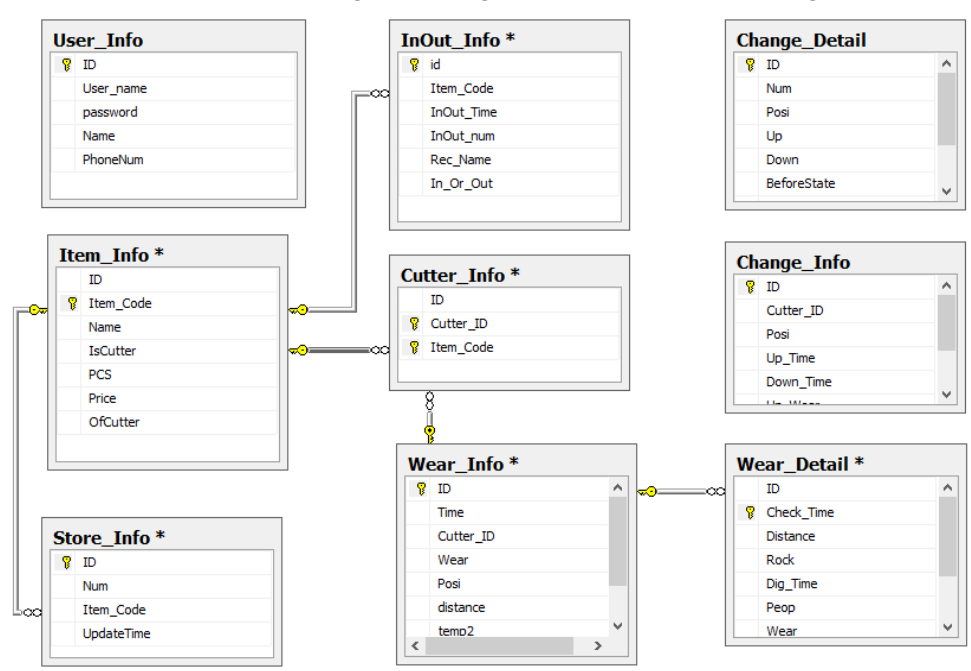

Fig. 3: Database diagram

Database connection is the basis for the realization of the entire system functions, so the need to complete the function before the completion of the database connection configuration. The database connection using ADO.NET technology, we first need to define the connection information database in the Web.Config file, including server address, database name the account password, etc:

<appSettings>

$<$ add key="ConnectionString"

value $="$ Server=(localhost $) ;$ database=CutterManageSys;uid=sa;pid=sa;Integrated security $=$ true"/>

$<$ /appSettings>

The definition of database connection class DbHelperSQL.cs, user-defined correlation method to execute SQL statements in this category. In the original reference should contain the System.Configuration namespace, and use PubConstant.cs to read Web.Config in the database connection string.

\section{Application Example -- TBM Cutter Information Management System of a Water Supply Project in Jilin}

\subsection{Project overview}

Jilin water supply general trunk tunnel length $110.0 \mathrm{~km}$, the main body of artesian water tunnel, water supply project total length $69.855 \mathrm{~km} .4$ standard construction section of trunk line length $22.955 \mathrm{~km}$, tunnel using TBM tunneling, drilling and blasting method by TBM, the length of $20.198 \mathrm{~m}$. 7KM section excavation limestone cave, weak TBM of excavation section, complex geology, construction management of large span, long construction time in winter. Through this system we have statistics from March 24, 2015 to January 5, 2016 the TBM cutter wear down Records, from March 27, 2015 to June 13, 2015 for the knife record. 


\subsection{System development environment}

Operating system development and operation of the system of the current mainstream Microsoft Windows 7 operating system. The deployment and operation of the system in the future can choose Win XP, Win2000 windows and server Microsoft IIS 7 series.Web server selection test client using Chrome browser, select the Microsoft InterNET Explorer client after the deployment of more than 6 versions of the database select Microsoft SQL Server 2008R2, system development cutters choose Visual Studio 2010.

\subsection{System display}

TBM cutter management information system functions include basic information, real-time information management, basic management, cutter wear statistics, cutter replacement statistics, as shown in Fig. 4 is the system login screen.

The basic information includes the basic information of the cutter, the ledger information and the tunneling parameters. The basic information of the cutter head is shown in Fig. 5, including the radius of the cutter and the angle of installation and the drawing of the cutter head.

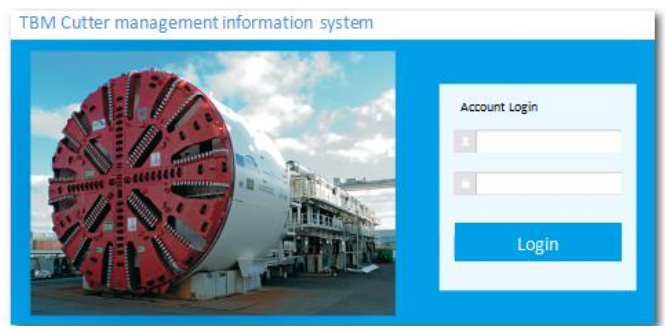

Fig. 4: System login interface

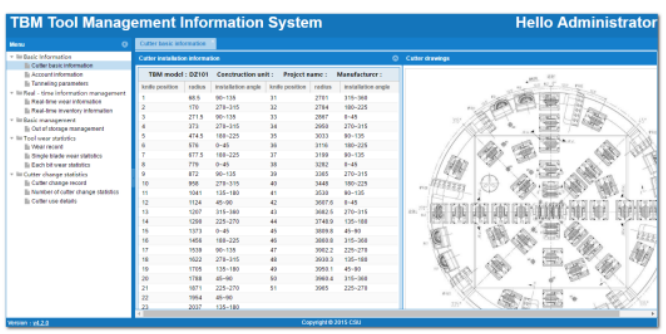

Fig. 5: Cutter basic information

Real time information management is mainly to provide users with real-time information and inventory cutter wear. Cutter wear refers to the last check knife, cutter wear on each of the hob value. Through this module can query the disc cutter wear value, the alarm on the cutter close to the wear limit; at the same time it can real-time inventory check the number of all parts, the alarm will run out of parts.

The basic management for the cutter inventory information out of storage management, in addition to the basic additions and deletions to change the search function, when you need to be able to export the results to the excel table.

Including the hob wear detection records of the results of the statistical cutter wear, wear information query, the statistics of single hob from installation to failure wear and each cutter wear amount of statistics, it is convenient for management personnel to directly see the wear trend of each cutter on the hob, the hob wear trend and single. As shown in Fig. 6 is the TBM cutter wear records from March 24, 2015 to January 5, 2016, click any recording time view details you can view detailed information and statistics of wear, wear pole, as shown in Fig. 7 Fig. 8 shows the wear statistics for each bit in this time period.

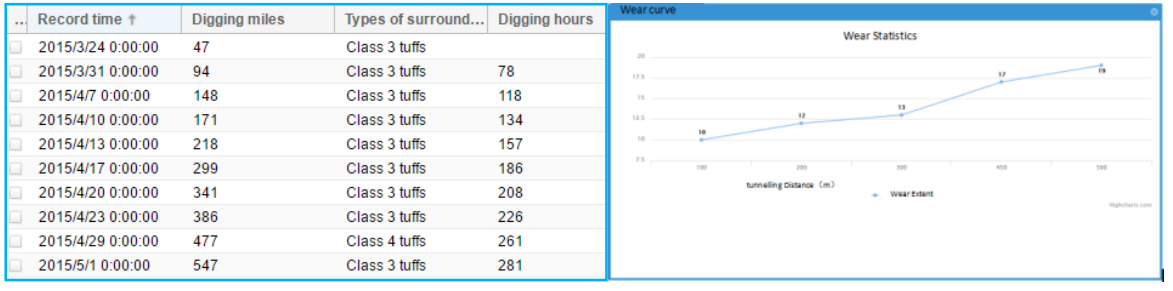

Fig. 6: Cutter wear records
Fig. 7: Single cutter wear curve statistics

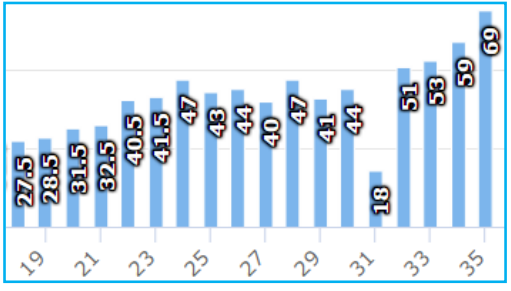

Fig. 8: Cutter wear statistics

The main statistical cutter change records and statistical cutter change information. Understanding each knife cutter change quantity, thus further analysis cutter change. At the same time whether the record detailed information cutter, for analysis on the service life of the cutter. As shown in Fig. 9 for the March 27, 2015 to June 13, 20154 months. Click on the record changer generation of cutter change the number of charts, displays the number of single day ATC ATC histogram and cumulative number of the line graph in 
cutter changing the number of charts will move the mouse to the corresponding time will show specific cutter change time and number, as shown in Fig. 10.

\begin{tabular}{|c|c|c|c|c|c|c|}
\hline Replacement staff & & & Change time : & & 鲷 & \\
\hline Change into the kn & nife number & & $\mathrm{Cha}$ & nge the knife numb & ber : & \\
\hline a Search for & 9 Add & enerate a chart & & & & \\
\hline Cutter chang.. & Number & Replacemen... & Position & Change into ... & Change the ... & \\
\hline $2015 / 3 / 270 \ldots$ & 51 & & $1,2,3,4,5,6, \ldots$ & & & \\
\hline $2015 / 4 / 230 \ldots$ & 5 & - & $16,48,49,50 \ldots$ & p $4239, p 425 \ldots$ & $\mathrm{p} 4252, \mathrm{p} 423 \ldots$ & - \\
\hline $2015 / 5 / 50 \ldots$ & 2 & - & 6,8 & $01773,0177 \ldots$ & p4214_.p42... & • \\
\hline $2015 / 5 / 170 \ldots$ & 5 & - & $47,48,49,50 \ldots$ & $668,669,66 \ldots$ & $\mathrm{p} 4246, \mathrm{p} 425 \ldots$ & • \\
\hline $2015 / 5 / 190 \ldots$ & 6 & - & $35,42,43,44 \ldots$ & $\mathrm{p} 4252, \mathrm{p} 425 \ldots$ & $\mathrm{p} 4235, \mathrm{p} 423 \ldots$ & • \\
\hline $2015 / 5 / 240 \ldots$ & 3 & - & $40,41,42$ & $\mathrm{p} 4246,663, \ldots$ & $\mathrm{p} 4240, \mathrm{p} 424 \ldots$ & • \\
\hline $2015 / 5 / 280 \ldots$ & 16 & - & $32,33,34,35 \ldots$ & $660,691,68 \ldots$ & $\mathrm{p} 4220, \mathrm{p} 425 \ldots$ & • \\
\hline $2015 / 6 / 10 \ldots$ & 5 & - & $47,48,49,50 \ldots$ & $666,002,00 \ldots$ & $673,666,68 \ldots$ & • \\
\hline $2015 / 6 / 20 \ldots$ & 1 & - & 23 & 667 & p4231 & • \\
\hline 2015/6/3 0.... & 7 & - & $24,25,26,27 \ldots$ & $676,677,68 \ldots$ & $\mathrm{p} 4232, \mathrm{p} 422 \ldots$ & • \\
\hline
\end{tabular}

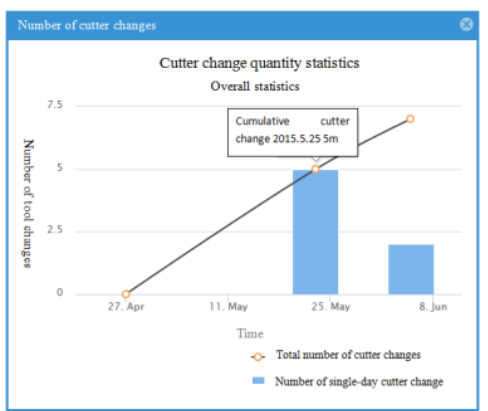

Fig. 9: Cutter change record

Fig. 10: The number of cutters for the exchange of local detail diagram

\section{Conclusion}

This paper designs the TBM tool information management system based on ASP.NET, and discusses its feasibility from system architecture, system function module design and database design. Finally a water supply project as an example, demonstrate the application effect of the system. The main conclusions are:

(1) The application of three layer B/S architecture has a good scalability and versatility, and can be easily and flexibly to join a variety of data analysis functions, and applies to a variety of engineering structure of the state monitoring;

(2) According to the different needs of the project, the system can choose a TBM as an object, but also can be used for the management of multiple TBM;

(3) The system can be posted to the server, the user can through the network remote access system. After testing, the system has the complete development, simple operation, and can meet the needs of users. Improve tool management efficiency and can accumulate a lot of engineering data for future analysis;

(4) At present, the system has carried on the basic statistics and the monitoring to the data processing. The future can be combined with the relevant algorithm to analyze the relationship between cutter wear and geological conditions and excavation parameters. Provide guidance on tool life prediction and cutter change.

\section{Acknowledgements}

This project is with Foundation item: 863 (2012AA041801); Hunan province science and technology major project (2014FJ1002); Hunan province strategic emerging industry science and technology research project (2015GK1029).

\section{References}

[1] Roby J, Sandell T, Kocab J, et al. The current state of disc cutter design and development directions[C]. North American Tunneling 2008 Proceedings, San Francisco, USA, 2008:36-45.

[2] Wang Mengshu. Development of Chinese railway, tunnel and underground space [J]. tunnel construction, 2010,30 (4):351-364.

[3] Fawcett D F, Remington R, Chapman P J. TBM drive management by use of computerized systems[M]. Tunnelling 94. Springer US, 1994:443-466.

[4] Wu Jie, Yu Jin Yu, Zhang Jinhui, et al. Design and application of structural monitoring information management system based on big data [J]. Journal of Hunan University, 2016,43 (9):76-81.

[5] Ren Jun Xinjiang Osaka tunnel engineering TBM. The construction of the integrated management information system construction and management of water conservancy and [J]., 2008,28 (1):40-42.

[6] Okubo S, Fukui K, Chen W. Expert system for applicability of tunnel boring machines in japan[J]. Rock Mechanics \& Rock Engineering, 2002, 36(4):305-322.

[7] Huang Xingrong. Design and implementation of three layer architecture based on B/S architecture [J]. computer knowledge and technology, 2015 (32):52-53. 